\title{
21. ELEMENTAL PROFILE OF IRIDIUM AND OTHER ELEMENTS NEAR THE CRETACEOUS/TERTIARY BOUNDARY IN HOLE 577B 1
}

\author{
Helen V. Michel and Frank Asaro, Lawrence Berkeley Laboratory \\ Walter Alvarez, University of California, Berkeley \\ and \\ Luis W. Alvarez, Lawrence Berkeley Laboratory²
}

\begin{abstract}
An Ir anomaly of $61 \mathrm{ng} / \mathrm{cm}^{2}$ was found in Deep Sea Drilling Project Hole 577B at the same stratigraphic level as the Cretaceous/Tertiary boundary defined by nannoplankton. This close correspondence supports the asteroid-impact theory for the Cretaceous/Tertiary boundary extinctions.
\end{abstract}

\section{INTRODUCTION}

An iridium anomaly associated with the Cretaceous/ Tertiary boundary has been identified at more than 50 sites worldwide (Alvarez et al., 1984). The most viable explanation for this worldwide anomaly is that an asteroid or other extraterrestrial body about $10 \mathrm{~km}$ in diameter impacted the Earth 65 m.y. ago, exploded, and distributed a dust cloud of terrestrial and extraterrestrial material which encircled the Earth and settled in a few months. Samples from Hole 577B were examined using neutron-activation analysis (NAA) to test this prediction and to search for other geochemical anomalies that would shed light on the mechanisms of boundary deposition.

A single core containing seven sections and a core catcher was taken at Hole 577B and an intact Cretaceous/Tertiary boundary was recovered. The sediment is an undeformed white to light brown nannofossil ooze (see Site 577 chapter, this volume). The boundary region is light brown (in the midst of a slightly whiter region); this colored region extends from about Sample 577B-1-4, $58 \mathrm{~cm}$ to $577 \mathrm{~B}-1-4,72 \mathrm{~cm}$. This boundary area is slightly firmer and more clay rich than the surrounding whiter regions.

\section{METHODS}

Continuous 1-cm samples were taken from 577B-1-4, $50 \mathrm{~cm}$ to $577 \mathrm{~B}-1-4,85 \mathrm{~cm}$. Above and below this interval, spot samples were taken about every $5 \mathrm{~cm}$. The sampling interval was increased farther from the boundary, eventually to a $50-\mathrm{cm}$ interval in Sections 1 and 6 of Core 577B-1.

A total of 77 samples were first dried at $110^{\circ} \mathrm{C}$ and then measured by high-precision methods of NAA (Perlman and Asaro, 1969). Iridium was calibrated against the Danish boundary clay standard "DINO-1" (Alvarez et al., 1982a). Calcium was calibrated against a primary standard, $\mathrm{CaCO}_{3}$. All other elements were calibrated against "Standard Pottery" (Perlman and Asaro, 1971).

\footnotetext{
${ }^{1}$ Heath, G. R., Burckle, L. H., et al., Init. Repts. DSDP, 86: Washington (U.S. Govt. Printing Office).

2 Addresses: (Michel, Asaro, L. Alvarez) Lawrence Berkeley Laboratory, University of California, Berkeley, CA 94720; (W. Alvarez) Department of Geology and Geophysics, University of California, Berkeley, CA 94720.
}

The data from the NAA are shown in Table 1. The listed errors are one sigma values of the counting error or $1 \%$, whichever is larger, and are about equal to the precision of measurement.

The iridium near the boundary (Samples 577B-1-4, $56 \mathrm{~cm}$ to 577B$1-4,77 \mathrm{~cm}$ ) could be measured precisely without any postirradiation chemistry. Farther from the boundary (as the level of Ir dropped), high temperature radiochemical separations were made in order to purify the Ir fraction without adding Ir carrier. In our procedure (which was inspired by the work of Rammensee and Palme, 1982) the same samples used for the regular NAA measurements (which each contained $100 \mathrm{mg}$ of rock and $50 \mathrm{mg}$ of cellulose binder) were heated to $1000^{\circ} \mathrm{C}$ in an oxidizing atmosphere to remove $\mathrm{CO}_{2}$ and oxidizable carbon. The residues were then each mixed in a boron nitride crucible with $200 \mathrm{mg}$ of powdered $\mathrm{Fe}, 100 \mathrm{mg}$ of Hawaiian basalt (which served as a silicate flux), and a few milligrams of graphite (which provided a reducing environment). The crucibles (which were covered with a graphite plate) were slowly brought up to a temperature of $1477^{\circ} \mathrm{C}$ in a Deltech furnace under an argon atmosphere. The furnace was held at that temperature for $1 \mathrm{hr}$. and then slowly cooled. The slow speed was necessary to prevent the mulite dome, which permitted the controlled atmosphere, from fracturing.

The iron fraction in each crucible was in the form of a ball imbedded in a silicate glass. Each crucible was crushed, and the Fe ball was removed and then cleaned of residual glass with pliers. In the oxidizing firing step, the yield of $\mathrm{Ir}$ and other elements was $99 \pm 1 \%$. In the reduction firing step the yields were $100.0 \pm 0.2,99.85 \pm 0.01$, and $98.6 \pm 0.2 \%$ for $\mathrm{Ir}, \mathrm{Co}$, and Fe radioactivities, respectively. Only 0.1 to $1 \%$ of the lithophile elements in the whole rock samples was extracted with the iron fraction.

This procedure provides a quantitative method of separating Ir radioactivity from the intense lithophile element radiations produced in NAA without adding Ir carrier, which can enhance the risk of laboratory contamination with Ir. The limits of detection for Ir were lowered to $\sim 0.015 \mathrm{ppb}$ for each sample, with a 1-day measurement time. As these low concentrations were considered to be background level Ir, four or more samples were analyzed simultaneously, resulting in a detection limit of $0.007 \mathrm{ppb}$ in a 1-day measurement for highly calcareous samples.

\section{RESULTS}

\section{Iridium Abundances}

The Ir abundance profile at Hole 577B is plotted as a function of stratigraphic position in Figure 1. One major peak is seen (in Section 4), along with five smaller ones. To determine which of these peaks is most likely associated with an impacting meteorite and which with the normal iridium background resulting from meteorit- 
Table 1. Elemental abundances in Core 577B-1.

\begin{tabular}{|c|c|c|c|c|c|c|c|c|c|c|c|c|c|}
\hline Section & $\begin{array}{c}\text { Interval } \\
(\mathrm{cm})\end{array}$ & Al $(\%)$ & $\mathrm{Ca}(\%)$ & Dy $(\mathrm{ppm})$ & $\mathrm{Mn}(\mathrm{ppm})$ & $\mathrm{U}(\mathrm{ppm})$ & $\mathrm{Ba}(\mathrm{ppm})$ & Ir $(p p b)$ & $\operatorname{Cr}(\mathrm{ppm})$ & Th $(\mathrm{ppm})$ & $\mathrm{Ni}(\mathrm{ppm})$ & $\mathrm{Tb}(\mathrm{ppm})$ & $\mathrm{Fe}\left(\%_{0}\right)$ \\
\hline \multirow{5}{*}{1} & $10-11$ & $0.33 \pm 0.02$ & $37.21 \pm 0.83$ & $9.22 \pm 0.11$ & $865 \pm 9$ & $0.241 \pm 0.020$ & $700 \pm 18$ & & $2.2 \pm 0.4$ & $0.97 \pm 0.03$ & $21 \pm 4$ & $1.457 \pm 0.044$ & $0.25 \pm 0 . c$ \\
\hline & $50-51$ & $31 \pm 0.03$ & $.72 \pm 0.88$ & $8.95 \pm 0.11$ & $834 \pm 8$ & $0.232 \pm 0.020$ & $960 \pm 21$ & \multirow{4}{*}{$0.039 \pm 0.008$} & & & & $1.419 \pm 0.043$ & \\
\hline & $87-88$ & $42 \pm 0.04$ & $40.51 \pm 0.94$ & $8.52 \pm 0.11$ & $897 \pm 9$ & $221 \pm 0.019$ & $884 \pm 20$ & & $3.8 \pm 0.4$ & & & 1.324 & \\
\hline & $00-101$ & $21 \pm 0.05$ & $39.12 \pm 0.95$ & $7.09 \pm 0.10$ & $747 \pm 7$ & $193=0.018$ & $723 \pm 18$ & & & $0.79 \pm$ & & $1.170 \pm$ & 0.20 \\
\hline & $140-141$ & $0.46 \pm 0.08$ & $36.80 \pm 0.99$ & $13.73 \pm 0.14$ & $1104 \pm 11$ & $.376 \pm 0.023$ & $656 \pm 19$ & & & $1.24 \pm$ & & $2.273 \pm$ & 0.34 \\
\hline \multirow{4}{*}{2} & $10-11$ & $0.43 \pm 0.02$ & $36.70 \pm 0.83$ & $3.25 \pm 0.13$ & $1066 \pm 11$ & $0.294 \pm 0.023$ & $662=19$ & \multirow{4}{*}{$0.073 \pm 0.009$} & & 1.15 & & $2.137 \pm$ & 0.31 \\
\hline & $31-32$ & $55 \pm 0.03$ & $7.82 \pm 0.88$ & $3.70 \pm 0.15$ & $1342 \pm 13$ & $.339=0.022$ & $624 \pm 19$ & & & $1.37 \pm$ & & $2.179 \pm$ & 0.43 \\
\hline & & $0.34 \pm 0.04$ & $37.43 \pm 0.89$ & $7.20 \pm 0.10$ & $707 \pm 7$ & $0.167 \pm 0.017$ & $514 \pm 15$ & & $2.3 \pm 0.3$ & $0.72 \pm$ & 22 & $1.165 \pm$ & 0.20 \\
\hline & $|20-12|$ & $0.25 \pm 0.05$ & $35.78 \pm 0.92$ & $6.99 \pm 0.10$ & $718 \pm 7$ & $0.172 \pm 0.017$ & $528 \pm 15$ & & $2.0 \pm 0.3$ & $0.58 \pm$ & & $1.076 \pm 0.034$ & $0.19 \pm$ \\
\hline & $20-21$ & $0.19 \pm 0.06$ & $8.40 \pm 0.97$ & $5.18 \pm 0.08$ & $241 \pm 2$ & $099 \pm 0.016$ & $312 \pm 12$ & & & 0.40 & & $0.783 \pm 0.025$ & $0.09=$ \\
\hline & $40-41$ & $0.10 \pm 0.02$ & $38.86 \pm 0.84$ & $4.73 \pm 0.07$ & $181 \pm 2$ & $0.113 \pm 0.015$ & $358 \pm 13$ & & & 0.29 & & $0.737 \pm 0.024$ & 0.06 \\
\hline & $60-61$ & $0.02 \pm 0.02$ & $37.24 \pm 0.84$ & $3.93 \pm 0.06$ & $124 \pm 1$ & $.081=0.014$ & $350 \pm 12$ & & $0.7 \pm 0.2$ & $0.26=$ & $5 \pm 3$ & $0.627 \pm 0.021$ & 0.05 \\
\hline 3 & $80-81$ & $0.08 \pm 0.03$ & $37.58 \pm 0.87$ & $5.12 \pm 0$ & $147 \pm 1$ & $09 \pm 0.6$ & $368 \pm 13$ & $0.016 \pm 0.006$ & & 0.35 & & $0.820 \pm 0.026$ & $0.07=$ \\
\hline & $100-101$ & $0.09 \pm 0.05$ & $37.59 \pm 0.93$ & $6.36 \pm 0.08$ & $233 \pm 2$ & $100 \pm 0.017$ & $229 \pm 13$ & & & 0.49 & $9 \pm 3$ & $1.038=0.032$ & $0.10=$ \\
\hline & $120-121$ & $0.14 \pm 0.06$ & $37.48=0.97$ & $4.78 \pm 0.07$ & $177 \pm 2$ & $.086 \pm 0.016$ & $335 \pm 13$ & & & 0.36 & $10 \pm 3$ & $0.787 \pm 0.025$ & 0.07 \\
\hline & $140-141$ & $0.08 \pm 0.02$ & $38.21 \pm 0.86$ & $4.28 \pm 0.07$ & $195 \pm 2$ & $.134 \pm 0.016$ & $157 \pm 11$ & & & & & $0.709 \pm 0.023$ & 0.08 \\
\hline & $1-2$ & $0.11 \pm 0.02$ & $.98 \pm 0.90$ & $4.14 \pm 0.07$ & $156 \pm 2$ & $123 \pm 0.015$ & $270 \pm 12$ & & & & & $0.633 \pm 0.021$ & 0.05 \\
\hline & $10-11$ & $0.07 \pm 0.03$ & $.79 \pm 0.92$ & $3.95 \pm 0.06$ & $118 \pm 1$ & $105 \pm 0.015$ & $328 \pm 12$ & $0.019 \pm 0$. & & & & $0.641 \pm 0.021$ & 0.05 \\
\hline & & $0.12 \pm 0.04$ & $37.84 \pm 0.94$ & $3.98 \pm 0.07$ & $129 \pm 1$ & $0.082 \pm 0.015$ & $350 \pm 12$ & & & 0.21 & $5 \pm 3$ & $0.621 \pm 0.021$ & 0.04 \\
\hline & $30-31$ & $0.14 \pm 0.06$ & $37.83 \pm 0.99$ & $3.58 \pm 0.07$ & $153 \pm 2$ & $0.079 \pm 0.014$ & $297 \pm 11$ & & & 0.16 & $9 \pm 2$ & $0.550 \pm 0.019$ & 0.04 \\
\hline & $35-36$ & $0.04 \pm 0.01$ & $36.52 \pm 0.8$ & $3.58 \pm 0.06$ & $90 \pm 1$ & $098 \pm 0.016$ & $254 \pm 11$ & $0.058 \pm 0.014$ & & 0.35 & $2 \pm 2$ & $0.512 \pm 0.018$ & 0.04 \\
\hline & $40-41$ & $0.07 \pm 0.01$ & $38.51 \pm 0$ & $3.83 \pm 0.08$ & $137 \pm 2$ & $.090 \pm 0.014$ & $267 \pm 12$ & $0.048 \pm$ & & & $8 \pm 2$ & $0.568 \pm 0.018$ & 0.03 \\
\hline & & $0.04 \pm 0.02$ & $39.36 \pm 0.92$ & $3.98 \pm 0.07$ & $99 \pm 1$ & $080 \pm 0.016$ & $278 \pm 11$ & 16 & & & & $0.555 \pm 0.019$ & 0.05 \\
\hline & & $0.07 \pm 0.01$ & $39.01=0.96$ & $4.26 \pm 0.08$ & $89 \pm 2$ & $093 \pm 0.013$ & $339 \pm 13$ & $0.110 \pm$ & & & & $0.633 \pm 0.020$ & 0.04 \\
\hline & & $0.04 \pm 0.03$ & $38.87 \pm 0.95$ & $4.51 \pm 0.07$ & $115 \pm 1$ & $085 \pm 0.016$ & & $0.194 \pm$ & & & $6 \pm 3$ & $0.585 \pm 0.020$ & 0.05 \\
\hline & & $0.03 \pm 0.04$ & $38.35 \pm 0.99$ & $4.85 \pm 0.07$ & $117 \pm 1$ & $169 \pm 0.016$ & & $0.272 \pm$ & & & $14 \pm 3$ & $0.715 \pm 0.024$ & 0.06 \\
\hline & & $0.10 \pm 0.05$ & $35.74 \pm 0.99$ & $4.40 \pm 0.07$ & $83 \pm 1$ & $280 \pm 0$. & & $0.166 \pm$ & & & & $0.648 \pm 0.022$ & 0.04 \\
\hline & & $0.07 \pm 0.01$ & $36.87 \pm 0.80$ & $5.36 \pm 0.07$ & $136 \pm 1$ & $121 \pm 0.017$ & $541 \pm 15$ & $0.261 \pm$ & & 0.18 & & $0.809 \pm 0.026$ & $0.08=$ \\
\hline & & $0.11 \pm 0.02$ & $38.78 \pm 1.00$ & $5.36 \pm 0.09$ & $163 \pm 2$ & $096 \pm 0.015$ & & $0.443 \pm$ & & & $13 \pm 3$ & $0.849 \pm 0.026$ & $0.09 \pm$ \\
\hline & & $0.11 \pm 0.02$ & $37.88 \pm 0$ & $5.84 \pm 0.07$ & $164 \pm 2$ & $111 \pm 0.018$ & & & & & 15 & $\begin{array}{l}0.863 \pm 0.028 \\
0.91 \pm 0.029\end{array}$ & $0.11=$ \\
\hline & 5 & $0.10 \pm 0.03$ & $39.02 \pm 0.89$ & $6.19 \pm 0.08$ & $183 \pm 2$ & $109 \pm 0.018$ & $660 \pm 16$ & & & & & $\begin{array}{l}0.901 \pm 0.029 \\
0.891 \pm 0.029\end{array}$ & $0.11 \pm$ \\
\hline & $58-59$ & $0.13 \pm 0.04$ & $39.77 \pm 0.92$ & $6.24 \pm 0.08$ & $191 \pm 2$ & $112 \pm 0.018$ & & $0.85 \pm$ & & & & $\begin{array}{l}0.891 \pm 0.029 \\
0.961 \pm 0.031\end{array}$ & $0.12 \pm$ \\
\hline & $59-60$ & $0.17 \pm 0.05$ & $37.54 \pm 0.94$ & $6.34 \pm 0.08$ & $169 \pm 2$ & $104 \pm 0.018$ & & & & 0.31 & $\begin{array}{l}15 \pm 3 \\
19 \pm 3\end{array}$ & $\begin{array}{l}0.901 \pm \\
0.985 \pm\end{array}$ & \\
\hline & $60-61$ & $0.14 \pm 0.03$ & $37.88 \pm 1.03$ & $6.38 \pm 0.10$ & & $132 \pm 0.016$ & & $1.68 \pm$ & & 0.45 & $\begin{array}{l}19 \\
20\end{array}$ & $1.001 \pm$ & $\begin{array}{l}0.19 \pm \\
0.20 \pm\end{array}$ \\
\hline & & $0.20 \pm 0.01$ & $37.68 \pm 0.79$ & $6.66 \pm 0.08$ & & $097 \pm 0.018$ & & & & & & $1.027 \pm$ & \\
\hline & $62-63$ & $0.22 \pm 0.02$ & $38.31 \pm 0.82$ & $6.80 \pm 0.08$ & & $107 \pm 0.017$ & & 2.74 & & & & $1.049 \pm$ & $\begin{array}{l}0.24 \pm \\
0.29 \pm\end{array}$ \\
\hline & & $0.28 \pm 0.05$ & $37.69 \pm 1.08$ & $6.50 \pm 0.10$ & & $140 \pm 0.016$ & & & & & & $1.057 \pm 0$ & $\begin{array}{l}0.29 \pm \\
0.38 \pm\end{array}$ \\
\hline & & $0.28 \pm 0.02$ & $36.26 \pm 0.88$ & & & & & & & & & $1.106 \pm 0$ & $\begin{array}{l}0.38 \pm 0 \\
0.46 \pm 0\end{array}$ \\
\hline & $\begin{array}{l}5-66 \\
-67\end{array}$ & $\begin{array}{l}0.28 \pm 0.02 \\
0.30 \pm 0.03\end{array}$ & $\begin{array}{l}35.01 \pm 0.90 \\
36.30 \pm 0.96\end{array}$ & $\begin{array}{l}6.80 \pm 0.09 \\
6.76 \pm 0.09\end{array}$ & $\begin{array}{l}253 \pm 3 \\
248 \pm 2\end{array}$ & 16 & $\begin{array}{l}828 \\
762\end{array}$ & $\begin{array}{l}4.55 \pm \\
4.75\end{array}$ & & 0 & & $1.066 \pm 0.032$ & $\begin{array}{l}0.46 \pm 0 \\
0.50 \pm 0\end{array}$ \\
\hline 4 & $7-68$ & $0.41 \pm 0.05$ & $35.35 \pm 0.99$ & $6.46 \pm 0.09$ & $285 \pm 3$ & 16 & & & & & & $1.023=0.031$ & $0.48 \pm 0$ \\
\hline${ }^{4}$ & 6 & $0.35 \pm 0.06$ & $35.18 \div 1.02$ & $6.43 \pm 0.09$ & & & & & & & & $0.977 \pm 0.029$ & $0.55 \pm$ \\
\hline & & $0.31 \pm 0.01$ & $36.59 \pm 0.73$ & $6.05 \pm 0.8$ & & & & & & & & \pm 0.030 & $0.54 \pm$ \\
\hline & -72 & $\begin{array}{l}0.33 \pm 0.02 \\
0.21 \pm 0.02\end{array}$ & $\begin{array}{l}37.51=0.80 \\
34.30 \div 0.78\end{array}$ & 5. & & & & & & & & $2 \pm 0.028$ & $0.53 \pm$ \\
\hline & & $\begin{array}{l}0.21 \pm 0.02 \\
0.24 \pm 0.03\end{array}$ & $\begin{array}{l}34.30 \pm 0.78 \\
35.84 \pm 0.83\end{array}$ & & & & & & & & & $6 \pm 0$. & $39 \pm 0$ \\
\hline & & $0.24 \pm 0.04$ & $\begin{array}{l}37.84 \pm 0.82 \\
37.62\end{array}$ & $\begin{array}{l}4.00 \pm 0 \\
3.86 \pm 0\end{array}$ & & & & & & & & & $0.46 \pm$ \\
\hline & & $0.15 \pm 0.01$ & $38.51 \pm 0.79$ & $\begin{array}{l}3.86 \pm 0 \\
3.72 \pm 0\end{array}$ & $\begin{array}{l}173 \\
115\end{array}$ & & $255 \pm 11$ & $\begin{array}{l}2.50 \pm \\
1.57 \pm\end{array}$ & & \pm & & & $\begin{array}{l}0.17 \pm 0 \\
0.10 \pm 0\end{array}$ \\
\hline & & $0.07 \pm 0.01$ & $39.39 \pm 0.82$ & $3.23 \pm 0$ & 158 & $\begin{array}{l} \pm 0.0 \\
\pm 0.0\end{array}$ & $248 \pm$ & $0.670 \pm$ & & 0. & & $\begin{array}{l}0.568 \pm 0.018 \\
0.477 \pm 0.016\end{array}$ & $\begin{array}{l}0.10 \pm 0.01 \\
0.05 \pm 0.01\end{array}$ \\
\hline & & $0.05 \pm 0.02$ & $38.79 \pm 0.84$ & $3.40 \pm 0$ & 102 & & & $0.601 \pm$ & & & & $4 \pm 0.016$ & $0.04 \pm 0.01$ \\
\hline & & $0.10 \pm 0.03$ & $38.32 \pm 0.88$ & $3.50 \pm 0$. & $120 \pm i$ & \pm 0.014 & & 1.0 & & & & $7 \pm 0.018$ & $0.08 \pm 0$ \\
\hline & & $03 \pm 0.04$ & $37.21 \pm 0.89$ & $3.16 \pm 0$. & $108 \pm 1$ & & & & & & & 16 & $5 \pm$ \\
\hline & & $3 \pm 0.02$ & $.14 \pm 0.86$ & $=0$ & & 4 & & & & & & \pm 0.017 & 0 \\
\hline & & $1 \pm 0.0$ & \pm 0.88 & $2 \pm 0$ & & \pm 0 & 289 & & & \pm & & \pm 0 & $4 \pm$ \\
\hline & & $0 \pm 0$ & 0 & \pm 0 & & +0 & & & & \pm & & $5 \pm 0$ & $8 \pm$ \\
\hline & & \pm 0 & & $29 \pm$ & i. & & & & & & & $8 \pm 0$ & $7 \pm$ \\
\hline & & \pm 0 & 39 . & $3.25 \pm$ & 113 & ( & & 0.5 & & \pm & & $5 \pm 0$ & $5 \pm$ \\
\hline & & \pm 0 & 40.3 & $2.99 \pm$ & 118 & c & & 0.2 & & \pm & & $7 \pm 0$ & \pm 0 \\
\hline & & \pm 0 & $40.45 \pm 0$. & $2.98 \pm$ & 112 & & & 0.2 & & \pm & & $5 \pm 0$ & \pm \\
\hline & & \pm 0 & $38.89 \pm 0.95$ & $2.92 \pm$ & 90 & 0 & & 0. & & \pm & & $2 \pm 0$ & $3 \pm$ \\
\hline & & \pm 0 & & $3.21 \pm 0$ & 105 & 0 & & \pm 0 & & & & $5 \pm 0$ & $3 \pm c$ \\
\hline & & 0 & & $3.52 \pm 0$ & $126 \pm$ & 0 & & $0.297 \pm 0.0$ & & & & $5 \pm 0$ & $6 \pm$ \\
\hline & & & & $3.42 \pm 0$ & 117 & \pm 0 & & & & & & \pm 0 & \pm \\
\hline & & & & $3.34 \pm 0$ & 131 & 0 & & & & 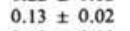 & & \pm 0 & \pm 0 \\
\hline & & & & $54 \pm 0$ & 154 & \pm & & $0.019 \pm 0.003$ & & t & & $\$ 0$ & \pm \\
\hline & & & & $9 \pm 0$ & 158 & \pm & & & & & & \pm 0 & 0 \\
\hline & & & & \pm 0 & & \pm & & & & & & \pm 0 & \\
\hline & & & & $6 \pm 0$ & & \pm 0 & & & & & & & \\
\hline & & & & $3.73 \pm 0$ & & \pm 0 & & $0.012=0.004$ & & & & 16 & \\
\hline 5 & & & & $4.22 \pm 0$ & 293 & \pm 0 & & & & & & & \\
\hline & & & & $62 \pm 0$ & 293 & \pm 0 & & & & & & 19 & \\
\hline & & & & $18.72 \pm 0$ & 2149 & $2 \pm 0$ & & $0.14 \pm 0.05$ & & & & \pm & \\
\hline & & & & $5.48 \pm 0$ & & \pm 0 & & & & & & 21 & \\
\hline & & $0.12 \pm$ & & $4.11 \pm 0$ & & \pm 0 & & & & & & & \\
\hline & & $0.16 \pm$ & & $4.29 \pm 0$ & & \pm 0 & 442 & $0.010 \pm 0.006$ & & & & 18 & \\
\hline 6 & & $0.18 \pm 0.01$ & & $.65 \pm 0.08$ & & $120 \pm 0$. & $323 \pm 9$ & & & $31 \pm 0$. & & $1 \pm 0.022$ & $.08 \pm 0$ \\
\hline & $140-141$ & $0.12 \pm 0.01$ & & $4.17 \pm 0.06$ & $158 \pm 2$ & $0.072 \pm 0.013$ & $224 \pm 8$ & & $0.6 \pm 0.2$ & $0.23 \pm 0.02$ & $4 \pm 2$ & $0.586 \pm 0.017$ & $0.04 \pm 0.0$ \\
\hline
\end{tabular}

Note: Asterisk indicates location of Cretaceous/Tertiary boundary as determined by nannofossil biostratigraphy.

ic dust, the following model was used: During any brief interval of geological time, both the clay deposition rate and the Ir deposition rate from meteorite dust in the area of Hole 577B would be approximately constant. The rate of $\mathrm{CaCO}_{3}$ deposition, however, could change as the biological productivity fluctuated or the calcite compensation depth varied. Changes in rates of $\mathrm{CaCO}_{3}$ deposition would affect the abundances of clay and iridium, but not their ratios. For the present analysis, Fe rather than $\mathrm{Al}$ was taken as a measure of the clay abundance because Fe was measured with comparably high sensitivity throughout the section studied while $\mathrm{Al}$ was not.

$\mathrm{Ir} / \mathrm{Fe}$ ratios are also shown in Figure 1. The background value is $2 \times 10^{-8}$ in both the Cretaceous and Tertiary regions even though the Ir abundance varies by an order of magnitude. Thus, the broad Ir peak in Sections 1 and 2 (Peak E, Fig. 1) and the relatively sharp peak in Section 5 (Peak A, Fig. 1) may have been caused simply by changes in the deposition rate of $\mathrm{CaCO}_{3}$. On the other hand, the main Ir peak and the three smaller 
Table 1. (Continued).

\begin{tabular}{|c|c|c|c|c|c|c|c|c|c|c|c|}
\hline Sm (ppm) & La (ppm) & Lu (ppm) & $\mathrm{Co}(\mathrm{ppm})$ & Sc (ppm) & $\mathrm{Sb}$ (ppm) & Eu (ppm) & $\mathrm{Ce}(\mathrm{ppm})$ & $\mathrm{Hf}(\mathrm{ppm})$ & Ta (ppm) & $\mathrm{Yb}(\mathrm{ppm})$ & $\mathrm{Nd}(\mathrm{ppm})$ \\
\hline $8.576 \pm 0.086$ & $34.76 \pm 0.67$ & $0.750 \pm 0.019$ & $10.15 \pm 0.19$ & $6.63 \pm 0.07$ & $0.17 \pm 0.05$ & $2.151 \pm 0.022$ & $18.28 \pm 0.50$ & $0.21 \pm 0.04$ & $0.043 \pm 0.003$ & $5.427 \pm 0.054$ & $40.1 \pm 1.1$ \\
\hline $8.430 \pm 0.084$ & $34.56 \pm 0.67$ & $0.716 \pm 0.018$ & $10.49 \pm 0.20$ & $6.05 \pm 0.06$ & $0.26 \pm 0.05$ & $2.389 \pm 0.024$ & $18.68 \pm 0.51$ & $0.29 \pm 0.04$ & $0.038 \pm 0.003$ & $5.246 \pm 0.053$ & $40.3 \pm 1.1$ \\
\hline $8.078 \pm 0.081$ & $33.15 \pm 0.66$ & $0.661 \pm 0.017$ & $11.46 \pm 0.21$ & $6.06 \pm 0.06$ & $0.22 \pm 0.05$ & $2.079 \pm 0.021$ & $19.75 \pm 0.50$ & $0.23 \pm 0.04$ & $0.044 \pm 0.003$ & $4.888 \pm 0.051$ & $39.0 \pm 1.1$ \\
\hline $6.659 \pm 0.067$ & $27.98 \pm 0.60$ & $0.560 \pm 0.015$ & $8.95 \pm 0.18$ & $5.44 \pm 0.05$ & $0.17 \pm 0.05$ & $1.736 \pm 0.017$ & $15.84 \pm 0.46$ & $0.27 \pm 0.04$ & $0.037 \pm 0.002$ & $4.071 \pm 0.044$ & $32.3 \pm 0.9$ \\
\hline $13.059 \pm 0.131$ & $50.77 \pm 0.84$ & $1.016 \pm 0.024$ & $14.17 \pm 0.24$ & $8.11 \pm 0.08$ & $0.25 \pm 0.06$ & $3.340 \pm 0.033$ & $27.30 \pm 0.59$ & $0.33 \pm 0.04$ & $0.062 \pm 0.003$ & $7.513 \pm 0.075$ & \\
\hline $12.609 \pm 0.126$ & $50.75 \pm 0.84$ & $0.995 \pm 0.023$ & $13.64 \pm 0.23$ & $7.92 \pm 0.08$ & $0.27 \pm 0.06$ & $3.202 \pm 0.032$ & $24.45 \pm 0.58$ & $0.39 \pm 0.04$ & $0.058 \pm 0.003$ & $7.387 \pm 0.074$ & $58.2 \pm 1.4$ \\
\hline $12.835 \pm 0.128$ & $49.78 \pm 0.83$ & $0.923 \pm 0.022$ & $16.29 \pm 0.26$ & $7.79 \pm 0.08$ & $0.28 \pm 0.06$ & $3.259 \pm 0.033$ & $28.05 \pm 0.58$ & $0.51 \pm 0.04$ & $0.067 \pm 0.003$ & $6.900 \pm 0.069$ & $58.3 \pm 1.4$ \\
\hline $6.891 \pm 0.069$ & $26.75 \pm 0.59$ & $0.520 \pm 0.014$ & $8.82 \pm 0.17$ & $4.30 \pm 0.04$ & $0.11 \pm 0.04$ & $1.752 \pm 0.018$ & $16.14 \pm 0.44$ & $0.17 \pm 0.03$ & $0.037 \pm 0.002$ & $3.846 \pm 0.042$ & $33.1 \pm 0.9$ \\
\hline $6.505 \pm 0.065$ & $26.06 \pm 0.58$ & $0.496 \pm 0.014$ & $9.06 \pm 0.17$ & $4.06 \pm 0.04$ & $0.15 \pm 0.04$ & $1.624 \pm 0.016$ & $14.82 \pm 0.43$ & $0.14 \pm 0.03$ & $0.034 \pm 0.002$ & $3.634 \pm 0.040$ & 30.7 \\
\hline $4.855 \pm 0.049$ & $22.98 \pm 0.53$ & $0.352 \pm 0.011$ & $3.03 \pm 0.10$ & $2.64 \pm 0.03$ & $0.11 \pm 0.03$ & $1.220 \pm 0.013$ & $6.81 \pm 0.34$ & $0.13 \pm 0.03$ & $0.022 \pm 0.002$ & $2.688 \pm 0.033$ & \pm 0.8 \\
\hline $4.167 \pm 0.042$ & $20.65 \pm 0.54$ & $0.329 \pm 0.010$ & $2.19 \pm 0.08$ & $2.48 \pm 0.02$ & $0.09 \pm 0.03$ & $1.064 \pm 0.012$ & $5.46 \pm 0.33$ & $0.08 \pm 0.03$ & $0.013 \pm 0.002$ & $2.483 \pm 0.031$ & $20.2 \pm 0.7$ \\
\hline $3.520 \pm 0.035$ & $20.20 \pm 0.52$ & $0.278 \pm 0.008$ & $1.61 \pm 0.07$ & $2.01 \pm 0.02$ & $0.13 \pm 0.03$ & $0.902 \pm 0.011$ & $3.90 \pm 0.30$ & $0.07 \pm 0.03$ & $0.009 \pm 0.002$ & $2.181 \pm 0.029$ & $17.4 \pm 0.6$ \\
\hline $4.782 \pm 0.048$ & $23.47 \pm 0.55$ & $0.340 \pm 0.010$ & $1.97 \pm 0.08$ & $2.61 \pm 0.03$ & $0.08 \pm 0.03$ & $1.223 \pm 0.013$ & $5.99 \pm 0.32$ & $0.08 \pm 0.03$ & $0.017 \pm 0.002$ & $2.623 \pm 0.032$ & $24.2 \pm 0.7$ \\
\hline $6.091 \pm 0.061$ & $28.13 \pm 0.63$ & $0.409 \pm 0.011$ & $2.92 \pm 0.09$ & $3.20 \pm 0.03$ & $0.08 \pm 0.03$ & $1.572 \pm 0.016$ & $9.03 \pm 0.37$ & $0.12 \pm 0.03$ & $0.026 \pm 0.002$ & $3.155 \pm 0.036$ & $30.4 \pm 0.9$ \\
\hline $4.699 \pm 0.047$ & $21.77 \pm 0.56$ & $0.322 \pm 0.010$ & $2.21 \pm 0.08$ & $2.53 \pm 0.03$ & $0.05 \pm 0.03$ & $1.220 \pm 0.013$ & $5.51 \pm 0.33$ & $0.08 \pm 0.03$ & $0.016 \pm 0.002$ & $2.553 \pm 0.032$ & $24.2 \pm 0.8$ \\
\hline $3.919 \pm 0.039$ & $18.74 \pm 0.55$ & $0.278 \pm 0.008$ & $2.52 \pm 0.09$ & $2.43 \pm 0.02$ & $0.09 \pm 0.03$ & $1.020 \pm 0.012$ & $5.48 \pm 0.33$ & $0.09 \pm 0.03$ & $0.016 \pm 0.002$ & $2.264 \pm 0.030$ & $19.8 \pm 0.7$ \\
\hline $3.745 \pm 0.037$ & $18.38 \pm 0.53$ & $0.272 \pm 0.008$ & $2.06 \pm 0.08$ & $2.19 \pm 0.02$ & $0.10 \pm 0.03$ & $0.965 \pm 0.011$ & $4.51 \pm 0.31$ & $0.07 \pm 0.03$ & $0.011 \pm 0.002$ & $2.141 \pm 0.029$ & $18.7 \pm 0.7$ \\
\hline $3.576 \pm 0.036$ & $19.25 \pm 0.52$ & $0.260 \pm 0.008$ & $1.74 \pm 0.07$ & $2.06 \pm 0.02$ & $0.05 \pm 0.02$ & $0.929 \pm 0.011$ & $4.06 \pm 0.29$ & $0.04 \pm 0.03$ & $0.012 \pm 0.002$ & $2.114 \pm 0.029$ & $17.3 \pm 0.7$ \\
\hline $3.515 \pm 0.035$ & $18.57 \pm 0.52$ & $0.262 \pm 0.008$ & $1.76 \pm 0.07$ & $2.00 \pm 0.02$ & $0.10 \pm 0.03$ & $0.907 \pm 0.011$ & $3.12 \pm 0.30$ & $0.03 \pm 0.03$ & $0.008 \pm 0.002$ & $2.108 \pm 0.027$ & $18.3 \pm 0.7$ \\
\hline $2.970 \pm 0.030$ & $17.38 \pm 0.49$ & $0.255 \pm 0.007$ & $1.98 \pm 0.08$ & $1.78 \pm 0.02$ & $0.07 \pm 0.03$ & $0.783 \pm 0.010$ & $3.17 \pm 0.28$ & $0.06 \pm 0.02$ & $0.009 \pm 0.002$ & $2.011 \pm 0.026$ & $14.8 \pm 0.6$ \\
\hline $2.910 \pm 0.029$ & $16.72 \pm 0.53$ & $0.260 \pm 0.010$ & $1.16=0.06$ & $1.67 \pm 0.02$ & $0.06 \pm 0.03$ & $0.762 \pm 0.010$ & $2.31 \pm 0.30$ & $0.01 \pm 0.03$ & $0.011 \pm 0.002$ & $2.028 \pm 0.030$ & $15.0 \pm 0.7$ \\
\hline $3.071 \pm 0.031$ & $17.84 \pm 0.43$ & $0.281 \pm 0.007$ & $1.70=0.07$ & $1.78 \pm 0.02$ & $0.09 \pm 0.03$ & $0.797 \pm 0.010$ & $2.28 \pm 0.26$ & $0.02 \pm 0.02$ & $0.012 \pm 0.002$ & $2.078 \pm 0.025$ & $16.1 \pm 0.5$ \\
\hline $3.062 \pm 0.031$ & $19.24 \pm 0.55$ & $0.273=0.010$ & $1.33 \pm 0.07$ & $1.72 \pm 0.02$ & $0.06 \pm 0.03$ & $0.769 \pm 0.010$ & $2.71 \pm 0.30$ & $0.05 \pm 0.03$ & $0.009 \pm 0.002$ & $2.144 \pm 0.031$ & $14.1 \pm 0.7$ \\
\hline $3.379 \pm 0.034$ & $20.36 \pm 0.44$ & $0.316 \pm 0.007$ & $1.29 \pm 0.06$ & $1.83 \pm 0.02$ & $0.07 \pm 0.03$ & $0.857 \pm 0.010$ & $2.42 \pm 0.26$ & $0.04 \pm 0.02$ & $0.010 \pm 0.002$ & $2.306 \pm 0.026$ & $17.5 \pm 0.5$ \\
\hline $3.551 \pm 0.036$ & $19.38 \pm 0.57$ & $0.320 \pm 0.011$ & $1.58 \pm 0.07$ & $1.88 \pm 0.02$ & $0.06 \pm 0.03$ & $0.900 \pm 0.011$ & $2.66 \pm 0.31$ & $0.07 \pm 0.03$ & $0.017 \pm 0.002$ & $2.336 \pm 0.033$ & $17.3 \pm 0.7$ \\
\hline $4.003 \pm 0.040$ & $22.71 \pm 0.60$ & $0.318 \pm 0.011$ & $1.58 \pm 0.07$ & $2.07 \pm 0.02$ & $0.10 \pm 0.03$ & $1.036 \pm 0.012$ & $3.35 \pm 0.32$ & $0.04 \pm 0.03$ & $0.016 \pm 0.002$ & $2.576 \pm 0.034$ & $20.5 \pm 0.8$ \\
\hline $3.601 \pm 0.036$ & $20.02 \pm 0.57$ & $0.306 \pm 0.011$ & $1.13 \pm 0.06$ & $1.90 \pm 0.02$ & $0.01 \pm 0.03$ & $0.956 \pm 0.012$ & $2.66 \pm 0.32$ & $0.07 \pm 0.03$ & $0.014 \pm 0.002$ & $2.387 \pm 0.033$ & $18.5 \pm 0.7$ \\
\hline $4.527 \pm 0.045$ & $24.89 \pm 0.64$ & $0.346 \pm 0.012$ & $1.82 \pm 0.08$ & $2.30 \pm 0.02$ & $0.05 \pm 0.03$ & $1.191 \pm 0.013$ & $3.16 \pm 0.34$ & $0.04 \pm 0.03$ & $0.015 \pm 0.002$ & $2.756 \pm 0.037$ & $24.3 \pm 0.9$ \\
\hline $4.785 \pm 0.048$ & $25.57 \pm 0.50$ & $0.383 \pm 0.010$ & $2.45 \pm 0.08$ & $2.44 \pm 0.02$ & $0.08 \pm 0.03$ & $1.231 \pm 0.013$ & $3.87 \pm 0.30$ & $0.03 \pm 0.03$ & $0.015 \pm 0.002$ & $2.819 \pm 0.031$ & $23.7 \pm 0.6$ \\
\hline $5.162 \pm 0.052$ & $27.42 \pm 0.66$ & $0.383 \pm 0.013$ & $2.72 \pm 0.10$ & $2.62 \pm 0.03$ & $0.08 \pm 0.03$ & $1.329 \pm 0.014$ & $4.01 \pm 0.36$ & $0.08 \pm 0.03$ & $0.017 \pm 0.002$ & $3.045 \pm 0.038$ & $27.1 \pm 0.9$ \\
\hline $5.406=0.054$ & $28.70 \pm 0.67$ & $0.381 \pm 0.013$ & $2.83 \pm 0.10$ & $2.78 \pm 0.03$ & $0.03 \pm 0.04$ & $1.402 \pm 0.015$ & $4.02 \pm 0.37$ & $0.04 \pm 0.03$ & $0.019 \pm 0.002$ & $3.126=0.039$ & $i \pm 0.9$ \\
\hline $5.409 \pm 0.054$ & $28.22 \pm 0.67$ & $0.398 \pm 0.014$ & $3.03 \pm 0.10$ & $2.80 \pm 0.03$ & $0.09 \pm 0.04$ & $1.387 \pm 0.014$ & $4.40 \pm 0.36$ & $0.16 \pm 0.03$ & $0.019 \pm 0.002$ & $3.107 \pm 0.039$ & $27.1 \pm 0.9$ \\
\hline $5.536 \pm 0.055$ & $29.35 \pm 0.69$ & $0.404 \pm 0.014$ & $2.81 \pm 0.10$ & $2.91 \pm 0.03$ & $0.12 \pm 0.04$ & $1.404 \pm 0.015$ & $4.44 \pm 0.37$ & $0.08 \pm 0.03$ & $0.017 \pm 0.002$ & $3.161 \pm 0.040$ & $27.4 \pm 1.0$ \\
\hline $5.671 \pm 0.057$ & $28.85 \pm 0.53$ & $0.417 \pm 0.011$ & $4.17 \pm 0.11$ & $3.09 \pm 0.03$ & $0.09 \pm 0.03$ & $1.483 \pm 0.015$ & $5.01 \pm 0.32$ & $0.14 \pm 0.03$ & $0.018 \pm 0.002$ & $189 \pm 0.034$ & $29.9 \pm 0.7$ \\
\hline $5.696 \pm 0.057$ & $29.73 \pm 0.69$ & $0.414 \pm 0.014$ & $4.40 \pm 0.12$ & $3.12 \pm 0.03$ & $0.10 \pm 0.04$ & $1.461 \pm 0.015$ & $4.39 \pm 0.37$ & $0.11 \pm 0.03$ & $0.022 \pm 0.002$ & $3.202 \pm 0.039$ & $29.2 \pm 1.0$ \\
\hline $5.775 \pm 0.058$ & $30.85 \pm 0.69$ & $0.414 \pm 0.014$ & $4.76 \pm 0.12$ & $3.19 \pm 0.03$ & $0.12 \pm 0.04$ & $1.466 \pm 0.015$ & $4.71 \pm 0.38$ & $0.16 \pm 0.03$ & $0.023 \pm 0.002$ & $3.233 \pm 0.040$ & $29.2 \pm 1.0$ \\
\hline $5.837 \pm 0.058$ & $31.58 \pm 0.55$ & $0.418 \pm 0.011$ & $6.18 \pm 0.14$ & $3.37 \pm 0.03$ & $0.15 \pm 0.04$ & $1.533 \pm 0.015$ & $5.77 \pm 0.33$ & $0.12 \pm 0.03$ & $0.024 \pm 0.002$ & $3.182 \pm 0.034$ & $29.6 \pm 0.7$ \\
\hline $6.264 \pm 0.063$ & $32.10 \pm 0.57$ & $417 \pm 0.011$ & $6.47 \pm 0.14$ & $3.61 \pm 0.04$ & $0.17 \pm 0.04$ & $1.601 \pm 0.016$ & $5.61 \pm 0.35$ & $0.22 \pm 0.03$ & $0.030 \pm 0.002$ & $9 \pm 0.034$ & 31.3 \\
\hline $6.361 \pm 0.064$ & $33.05 \pm 0.58$ & $435 \pm 0.011$ & $7.53 \pm 0.16$ & $3.68 \pm 0.04$ & $0.17 \pm 0.04$ & $1.638 \pm 0.016$ & $5.50 \pm 0.36$ & $0.20 \pm 0.03$ & $0.035 \pm 0.002$ & $7 \pm 0.034$ & 32.7 \\
\hline $6.086 \pm 0.061$ & $31.20 \pm 0.57$ & $0.396 \pm 0.011$ & $7.74 \pm 0.16$ & $3.64 \pm 0.04$ & $0.22 \pm 0.04$ & $1.568 \pm 0.016$ & $5.48 \pm 0.35$ & $0.17 \pm 0.03$ & $0.038 \pm 0.002$ & $12 \pm 0.033$ & 0.7 \\
\hline $5.789 \pm 0.058$ & $29.57 \pm 0.56$ & $0.394 \pm 0.011$ & $8.99 \pm 0.17$ & $3.56 \pm 0.04$ & $0.17 \pm 0.04$ & $1.477 \pm 0.015$ & $5.21 \pm 0.35$ & $0.24 \pm 0.03$ & $0.040 \pm 0.002$ & $8 \pm 0.032$ & 0.7 \\
\hline $5.689 \pm 0.057$ & $28.26 \pm 0.54$ & $0.372 \pm 0.010$ & $7.97 \pm 0.16$ & $3.39 \pm 0.03$ & $0.22 \pm 0.04$ & $1.442 \pm 0.014$ & $5.75 \pm 0.34$ & $0.31 \pm 0.03$ & $0.035 \pm 0.002$ & $2.793 \pm 0.032$ & 27.8 \\
\hline $5.476 \pm 0.055$ & $27.84 \pm 0.54$ & $0.362 \pm 0.010$ & $7.77 \pm 0.15$ & $3.10 \pm 0.03$ & $0.17 \pm 0.04$ & $1.383 \pm 0.014$ & $5.56 \pm 0.34$ & $0.22 \pm 0.03$ & $0.036 \pm 0.002$ & $2.762 \pm 0.031$ & \pm 0.7 \\
\hline $5.147 \pm 0.051$ & $26.42 \pm 0.53$ & $0.327 \pm 0.008$ & $6.55 \pm 0.14$ & $2.87 \pm 0.03$ & $0.23 \pm 0.04$ & $1.295 \pm 0.013$ & $4.70 \pm 0.33$ & $0.27 \pm 0.03$ & $0.036 \pm 0.002$ & $9 \pm 0.030$ & $26.2 \pm 0.6$ \\
\hline $3.495 \pm 0.035$ & $20.51 \pm 0.47$ & $0.275 \pm 0.007$ & $4.53 \pm 0.11$ & $2.48 \pm 0.02$ & $0.13 \pm 0.04$ & $0.892 \pm 0.011$ & $3.59 \pm 0.30$ & $0.15 \pm 0.03$ & $0.030 \pm 0.002$ & $8 \pm 0.025$ & 0.5 \\
\hline $3.498 \pm 0.035$ & $19.03 \pm 0.45$ & $0.278 \pm 0.007$ & $4.45 \pm 0.11$ & $2.10 \pm 0.02$ & $0.18 \pm 0.04$ & $0.893 \pm 0.011$ & $3.29 \pm 0.30$ & $0.18 \pm 0.03$ & $0.026 \pm 0.002$ & $2.151 \pm 0.025$ & $19.3 \pm 0.5$ \\
\hline $3.280 \pm 0.033$ & $18.70 \pm 0.44$ & $0.273 \pm 0.007$ & $4.21 \pm 0.10$ & $1.69 \pm 0.02$ & $0.09 \pm 0.03$ & $0.866 \pm 0.010$ & $2.99 \pm 0.26$ & $0.06 \pm 0.02$ & $0.019 \pm 0.002$ & $2.090 \pm 0.025$ & 17.1 \\
\hline $3.119 \pm 0.031$ & $17.71 \pm 0.44$ & $0.257 \pm 0.006$ & $2.67 \pm 0.08$ & $1.49 \pm 0.01$ & $0.17 \pm 0.03$ & $0.806 \pm 0.010$ & $2.14 \pm 0.27$ & $0.08 \pm 0.02$ & $0.014 \pm 0.002$ & $1.947 \pm 0.024$ & 0.5 \\
\hline $2.714 \pm 0.027$ & $16.01 \pm 0.40$ & $0.235 \pm 0.006$ & $2.88 \pm 0.08$ & $1.26 \pm 0.01$ & $0.07 \pm 0.03$ & $0.681 \pm 0.009$ & $2.04 \pm 0.24$ & $0.05 \pm 0.02$ & $0.014 \pm 0.002$ & $1.815 \pm 0.023$ & $14.0 \pm 0.5$ \\
\hline $2.803 \pm 0.028$ & $16.46 \pm 0.41$ & $0.243 \pm 0.006$ & $1.82 \pm 0.07$ & $1.28 \pm 0.01$ & $0.08 \pm 0.02$ & $0.711 \pm 0.009$ & $2.42 \pm 0.24$ & $0.02 \pm 0.02$ & $0.011 \pm 0.002$ & $1.866 \pm 0.023$ & $13.2 \pm 0.5$ \\
\hline $2.943 \pm 0.029$ & $16.16 \pm 0.43$ & $0.228 \pm 0.006$ & $2.09 \pm 0$. & $1.37 \pm 0$ & $0.02 \pm$ & $0.748 \pm 0.009$ & $2.52 \pm 0.26$ & $0.06 \pm 0.02$ & $0.014 \pm 0.002$ & $6 \pm 0.023$ & $14.9 \pm 0.5$ \\
\hline $2.729 \pm 0.027$ & $14.56 \pm 0.41$ & $0.228 \pm 0.006$ & $1.88 \pm 0$. & $1.28 \pm 0$ & $0.07 \pm$ & $0.681 \pm 0.0$ & $2.21 \pm 0.25$ & $0.05 \pm 0.02$ & $0.014 \pm 0.002$ & $5 \pm 0.022$ & $13.6 \pm 0.5$ \\
\hline $2.478 \pm 0.025$ & $14.73 \pm 0$ & $0.215 \pm 0.008$ & $1.59 \pm 0$. & $1.22 \pm 0$ & $0.04 \pm$ & $0.629 \pm 0$ & $1.82 \pm 0.27$ & $0.04 \pm 0.02$ & $0.012 \pm 0.002$ & $9 \pm 0.026$ & $0 \pm 0.7$ \\
\hline $2.761 \pm 0.028$ & $15.54 \pm 0$ & $21 \pm 0$. & $1.89 \pm 0$ & $1.25 \pm 0$ & $0.05 \pm$ & $0.697 \pm 0$ & $2.00 \pm$ & $0.01 \pm$ & $5 \pm 0.002$ & $8 \pm 0.026$ & $9 \pm 0.7$ \\
\hline $3.014 \pm 0.030$ & $16.43 \pm 0$ & $27 \pm 0$. & $2.25 \pm 0$ & $1.35 \pm 0$ & $0.07 \pm$ & $0.756 \pm 0$. & $2.35 \pm$ & $0.08 \pm$ & $0.011 \pm 0.002$ & $5 \pm 0.027$ & $8 \pm 0.7$ \\
\hline $2.869 \pm 0.029$ & $16.03 \pm 0$ & $0.226 \pm 0$. & $2.23 \pm 0$ & $1.26 \pm 0$ & $0.06 \pm$ & $19 \pm 0$. & $2.36 \pm$ & $0.05 \pm$ & $0.014 \pm 0.002$ & $1 \pm 0.026$ & $6 \pm 0.7$ \\
\hline $2.728 \pm 0.027$ & $14.78 \pm 0.50$ & $0.214 \pm 0$. & $1.59 \pm 0$. & $1.19 \pm 0$ & $0.39 \pm$ & $67 \pm 0$ & $2.56 \pm$ & .02 & $7 \pm 0.002$ & 025 & $4 \pm 0.7$ \\
\hline $2.534 \pm 0.025$ & $14.95 \pm 0$ & $98 \pm 0$. & $1.43 \pm 0$ & $1.21 \pm$ & $0.10 \pm$ & 0. & $1.97 \pm$ & .02 & \pm 0.002 & $9 \pm 0$ & $6 \pm 0.7$ \\
\hline & $13.68 \pm 0.50$ & $\infty 0 \pm 0$. & $1.44 \pm 0$ & $1.18 \pm 0$ & $0.02 \pm$ & 0.6 & $2.25 \pm$ & .02 & \pm 0.002 & $9 \pm 0$ & 50.7 \\
\hline \multirow[t]{18}{*}{$2.619 \pm 0.026$} & $15.24 \pm 0.51$ & $91 \pm 0 . C$ & $1.06 \pm 0$ & $1.19 \pm 0$ & $0.01 \pm$ & 0.6 & $1.84 \pm$ & .02 & \pm 0.002 & 25 & \\
\hline & & $0.192 \pm 0$. & $1.01 \pm 0$ & $1.13 \pm$ & $0.03 \pm$ & $0.644 \pm$ & $1.44 \pm$ & .02 & \pm 0.002 & $3 \pm 0$ & \\
\hline & & $0.215 \pm 0.0$ & $1.66 \pm 0$ & $1.14 \pm 0$ & $0.06 \pm 0$ & $0.784 \pm 0$ & $2.05 \pm$ & $8 \pm 0$ & \pm 0.002 & $6 \pm 0$ & \\
\hline & & $20 \pm 0.0$ & $1.22 \pm 0$ & $1.15 \pm 0$ & $0.01 \pm 0$ & $0.721 \pm 0$ & $2.62 \pm$ & $3 \pm 0.02$ & $2 \pm 0$ & $5 \pm 0$ & \\
\hline & & $s=0.0$ & $1.35 \pm 0$ & $1.25 \pm 0$ & $0 \pm$ & $1=0$ & $2.23 \pm$ & \pm 0 & \pm 0 & $4 \pm 0$ & \\
\hline & & $9 \pm 0$ & $1.51 \pm 0$ & $1.24 \pm 0$ & $2 \pm$ & $3 \pm 0$ & $3.06 \pm 0$ & \pm & \pm 0 & $0 \pm 0$ & 4 \\
\hline & & $6 \pm 0$. & $1.52 \pm 0$ & $1.28 \pm 0$ & $4 \pm$ & $2 \pm 0$. & $2.85 \pm 0$ & $6 \pm 0.02$ & $0.013 \pm 0$ & $i .781 \pm 0$ & \\
\hline & & $2 \pm 0$. & $1.55 \pm 0$ & $1.41 \pm 0$ & $0.09 \pm 0$ & $90 \pm 0.011$ & $2.74 \pm 0$ & $0.03 \pm 0.02$ & $0.013 \pm 0.002$ & $1.845 \pm 0$ & $18.4 \pm 0.5$ \\
\hline & & $7 \pm 0$ & $1.52 \pm 0$ & $1.40 \pm 0$ & $0.03 \pm$ & \pm 0. & $2.99 \pm$ & $0.03 \pm 0$ & $9 \pm 0.002$ & $5 \pm 0$ & $16.3 \pm$ \\
\hline & & $37 \pm 0$. & $2.24 \pm 0$ & $1.55 \pm 0$ & \pm & \pm 0.0 & $3.54 \pm$ & 02 & $4 \pm 0$ & $1.830 \pm 0$. & $16.8 \pm$ \\
\hline & & $0 \pm 0$. & $2.27 \pm 0$ & $1.73 \pm 0$ & $0.07 \pm$ & $9 \pm 0.0$ & 3.36 & \pm & $2 \pm 0$ & $2.121 \pm 0.024$ & $18.8 \pm 0$ \\
\hline & & $87 \pm 0.007$ & $2.75 \pm 0$. & $1.99 \pm 0.02$ & $0.08 \pm 0$ & $6 \pm 0.012$ & $4.58 \pm$ & \pm & 50 & $2.235 \pm 0.025$ & $21.9 \pm 0.5$ \\
\hline & & $5 \pm 0.0$ & $22.11 \pm 0$. & $6.00 \pm 0$. & $0.31 \pm 0$ & $6 \pm 0.055$ & $29.22 \pm$ & 04 & \pm 0 & $6.961 \pm 0.070$ & $106.6 \pm 16.0$ \\
\hline & & $8 \pm 0$. & $3.67 \pm 0$ & $2.07 \pm 0.02$ & $0.06 \pm 0$ & \pm 0.013 & 4.45 & 03 & $0 \pm 0$ & $2.467 \pm 0.027$ & \\
\hline & & \pm 0 . & $2.18 \pm 0$ & $1.59 \pm 0$ & $0.04 \pm 0$ & \pm 0.0 & 2. & \pm 0 & $2 \pm 0$ & 90.024 & \\
\hline & & \pm 0 . & $2.40 \pm 0$. & $1.58 \pm 0$ & $0.10 \pm$ & \pm 0.0 & \pm 0.26 & \pm 0 & $s \pm 0$. & $1.985 \pm 0$ & \\
\hline & & $16 \pm 0.008$ & $3.73 \pm 0.10$ & $1.82 \pm 0.02$ & $0.09 \pm 0.03$ & $1.283 \pm 0.013$ & $4.59 \pm 0.29$ & $0.09 \pm 0.03$ & $17 \pm 0.002$ & $2 \pm 0$. & $26.4 \pm 0.6$ \\
\hline & & $0.280 \pm 0.007$ & $1.69 \pm 0.07$ & $1.34 \pm 0.01$ & $0.04 \pm 0.02$ & $0.830 \pm 0.010$ & $2.61 \pm 0.25$ & $0.05 \pm 0.02$ & $0.012 \pm 0.002$ & $2.099 \pm 0.024$ & $16.5 \pm 0.4$ \\
\hline
\end{tabular}

peaks in Section 4 (Peak B at $110 \mathrm{~cm}$, Peak C at $81-83 \mathrm{~cm}$, Peak D at $77-78 \mathrm{~cm}$ ) have $\mathrm{Ir} / \mathrm{Fe}$ ratios that are one to two orders of magnitude higher than background (Fig. 1), and they should, therefore, have a different origin. The elevated tail in the lower part of Section 4 is also not likely to be due to background (Fig. 1).

The rise of the main Ir peak is steepest between Samples 577B-1-4, $74 \mathrm{~cm}$ and 577B-1-4, $73 \mathrm{~cm}$, which is in close agreement with the location of the Cretaceous/Tertiary boundary at 577B-1-4, $72 \mathrm{~cm}$ defined by nannofossil studies (Site 577 chapter, this volume; Monechi, this volume). Similar agreements between biostratigraphic and geochemically defined Cretaceous/Tertiary boundary levels (Asaro et al., 1982) have been found in many marine sections, and these would be expected from the asteroidimpact theory. The three smaller peaks below the main peak (Peaks B, C, and D in Fig. 1) and the elevated tail, however, should not be primary deposits in Cretaceous sediment if the asteroid-impact theory is correct.

Bioturbation can cause smearing of sharp boundaries, but it is not clear that it would produce the two well-shaped peaks (C and D) seen in Figure 1. Also the 


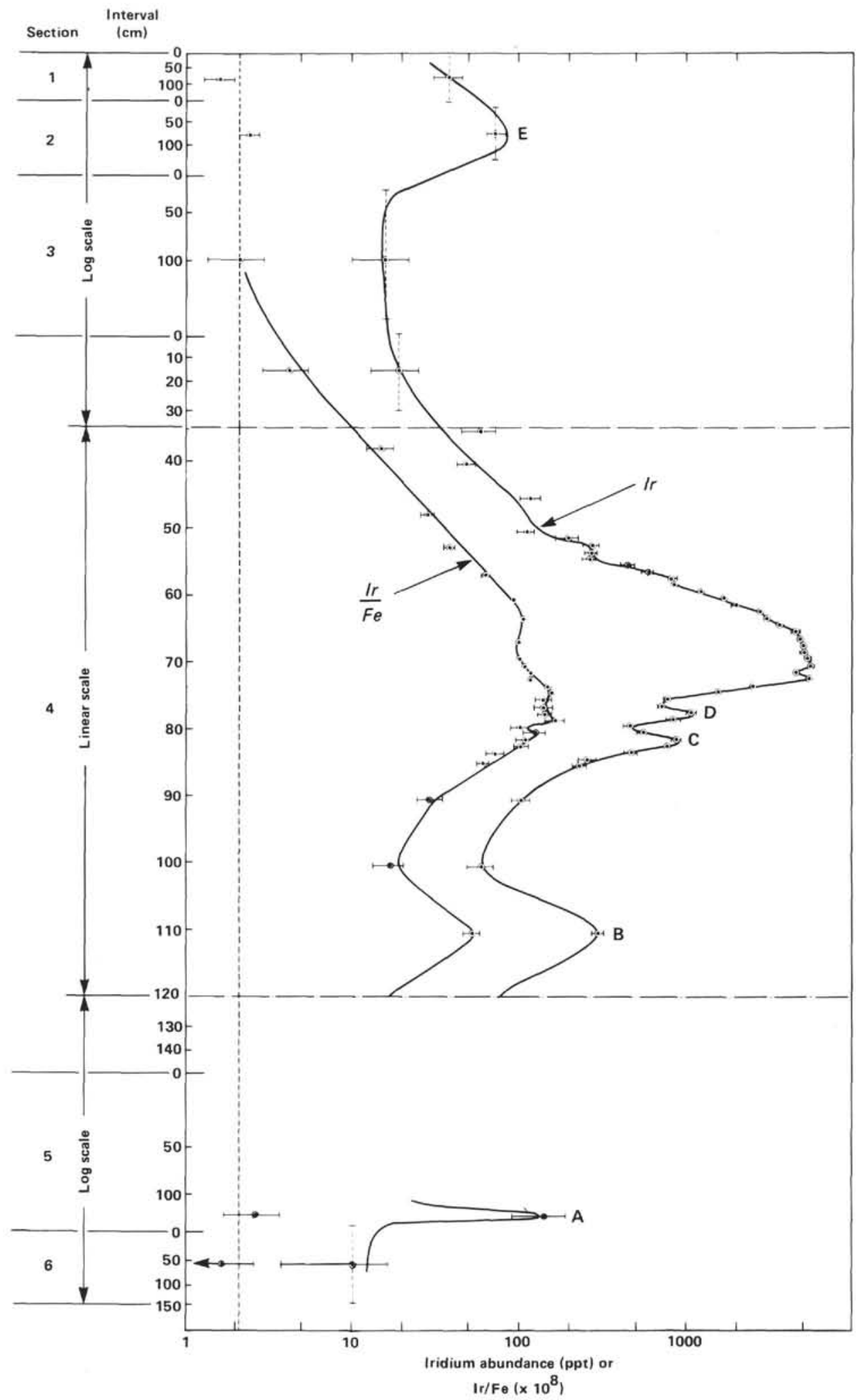

Figure 1. Stratigraphic position in Core 577B-1 versus abundance of iridium (ppt) and $(\mathrm{Ir} / \mathrm{Fe}) \times 10^{-8}$. The main Ir anomaly is located between 65 and $73 \mathrm{~cm}$ in Section 4 . Five smaller anomalies, labeled A-E occur in Samples 577B-1-5, 120-121 cm (Peak A) 577B-1-4, 110-111 cm (Peak B), 577B-1-4, 81$83 \mathrm{~cm}$ (Peak C), 577-1-4, 77-78 cm (Peak D) and 577B-1-1, $10 \mathrm{~cm}$ to 577B-1-2, $121 \mathrm{~cm}$ (Peak E). The depth over which a sample was collected for the Ir measurements is shown by a vertical bar. A dashed vertical bar indicates the collection was not continuous. 
extent of penetration of the tail into the Cretaceous sediment (over a meter) and the depth of Peak B below the main $\mathrm{Ir}$ distribution $(38 \mathrm{~cm})$ are greater than would be expected from bioturbation. Iridium in Cretaceous/Tertiary sediments is to some extent soluble in acids (L. W. Alvarez et al., 1980) and the observed tail may be due to mobility of the Ir. It is also possible that Peak B is due to contamination or to another impact (Davis et al., 1984, Whitmire and Jackson, 1984). A method of checking some of these suppositions would be to resample the material below the sliced surface. As neither bioturbation nor contamination is likely to reproduce Peaks B, $\mathrm{C}$, and $\mathrm{D}$ in measurements of such samples, another cause would be likely if the peaks are still observed.

\section{Siderophile Element Abundances}

Cobalt, $\mathrm{Ni}, \mathrm{Cr}$, and $\mathrm{Fe}$ all show abundance profiles similar to that of Ir (Table 1) near the Cretaceous/Tertiary boundary, as do the lithophile elements $\mathrm{Al}$ and $\mathrm{Ta}$ (Fig. 3). The Ni/Ir ratio is about a factor of 3 lower than the chondritic value and the $\mathrm{Cr} / \mathrm{Ir}$ ratio is about a factor of 1.5 lower.

At Stevns Klint, Denmark and at Deep Sea Drilling Project (DSDP) Hole 465A in the Central Pacific Ocean, siderophile elements measured have ratios consistent with mixtures of terrestrial and meteoritic (chondritic) components (F. Asaro, W. Alvarez, H. V. Michel, L. W. Alvarez, M. Kastner, and J. Thiede, unpubl. data). The anoxic environment in which sediments from these sections were deposited may have preserved the ratios of these siderophile elements, as these elements form insoluble sulfides.

Sulfur was not measured in the Hole 577B sediments. As none of the sediments contain measurable amounts of Se $(<0.7 \mathrm{ppm})$, which is normally associated with sulfide deposits, the environment of deposition at Site 577 was probably not anoxic, and some siderophile elements may have been lost during diagenesis.

\section{Rare-Earth Patterns}

Hole 577B rare-earth element abundance patterns divided by the chondritic values (Masuda et al., 1973) are shown in Figure 2. The large negative $\mathrm{Ce}$ anomaly (characteristic of seawater) indicates that the predominant rare-earth elements were originally dissolved in seawater. As seen in Figure 3, Ce (over and above the amount that is associated with $\mathrm{Sm}$ in the region directly above and below the boundary) has the same abundance profile as Ir in the boundary region. This excess Ce may be due to a detrital component from the impact site. In addition to excess $\mathrm{Ce}$, the ratio of heavy to light rare-earth elements is smaller in the Ir-rich region than above and below this region.

\section{DISCUSSION}

\section{Sedimentation Rate}

Barker and Anders (1968) measured the content of Ir in Pacific red clays as a maximum of $140 \mathrm{ppt}$, for a deposition rate of $1 \mathrm{~mm}$ per thousand years and a density of $0.5 \mathrm{~g}$ of solid per cubic centimeter of wet sediment.

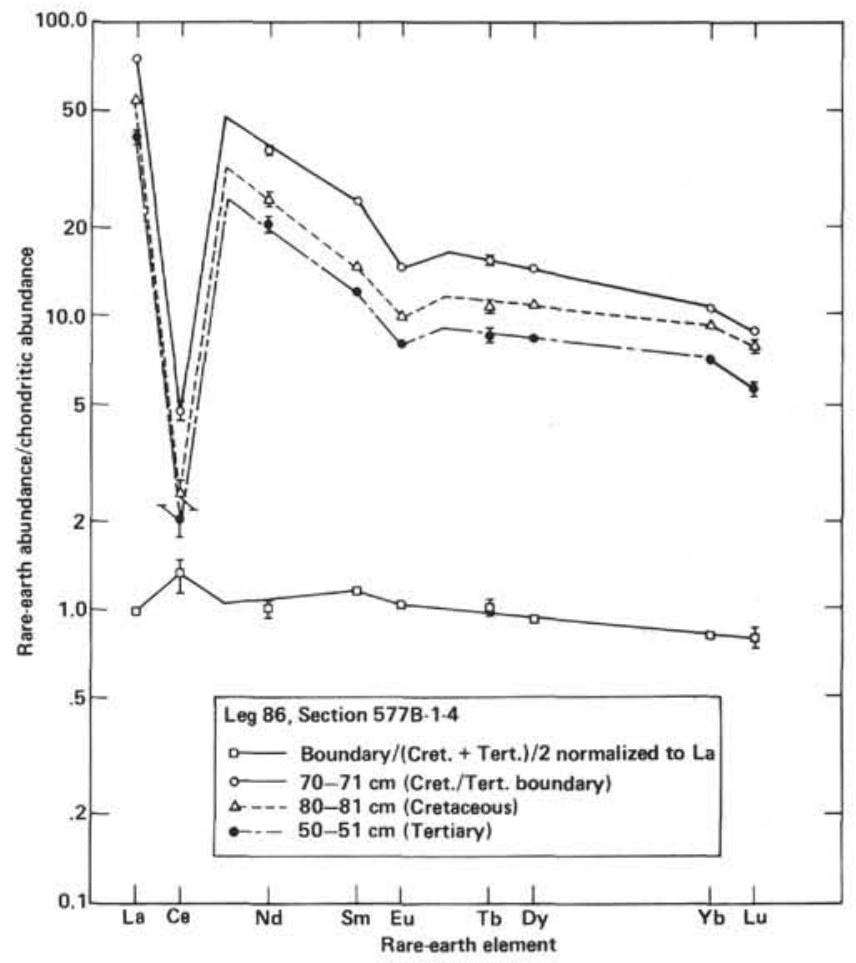

Figure 2. Rare-earth element abundances divided by those of Leedy chondrite (Masuda et al., 1973) as a function of atomic number.

About half of this Ir was attributed to an extraterrestrial source and half to sources independent of the deposition rate, but the uncertainties were such that all of the Ir could have come from the extraterrestrial source. $\mathbf{K y}$ te and Wasson (1982) found a somewhat higher abundance of Ir, when normalized to the same deposition rate. With the Barker and Anders (1968) maximum Ir deposition value, a $1.9 \mathrm{~m} / \mathrm{m}$.y. average sedimentation rate given in the Site 577 chapter (this volume) for the Cretaceous/Tertiary boundary region, and a density of $0.8 \mathrm{~g} /$ $\mathrm{cm}^{3}$, the background Ir should be about $46 \mathrm{ppt}$. This is consistent with the present work as it is about midway between the highest (73 ppt) and lowest (10 ppt) values of the measured Ir background. Assuming the Barker and Anders (1968) maximum meteorite Ir deposition rate and a density of $0.8 \mathrm{~g} / \mathrm{cm}^{3}$, the rate of sedimentation at any stratigraphic level in this section, where the "background" Ir abundance from normal meteoritic dust can be measured, would therefore be $46(\mathrm{~m} / \mathrm{m} . \mathrm{y}$.)/background Ir abundance (ppt).

\section{Integrated Amount of Ir}

If the products of the $\mathrm{Ir}$ abundance in each stratigraphic interval and the stratigraphic height of the interval are summed over the boundary region, and a background of $0.015 \mathrm{ppb}$ times the total stratigraphic height is removed, and the net value is multiplied by a density of $0.8 \mathrm{~g} / \mathrm{cm}^{3}$, there are found to be $61 \mathrm{ng} / \mathrm{cm}^{2}$ of anomalous $\mathrm{Ir}$ in the boundary region. This is comparable to the average amount found at other studied Cretaceous/ Tertiary sections worldwide $\left(50 \mathrm{ng} / \mathrm{cm}^{2}\right)$ (W. Alvarez et al., 1982b). 


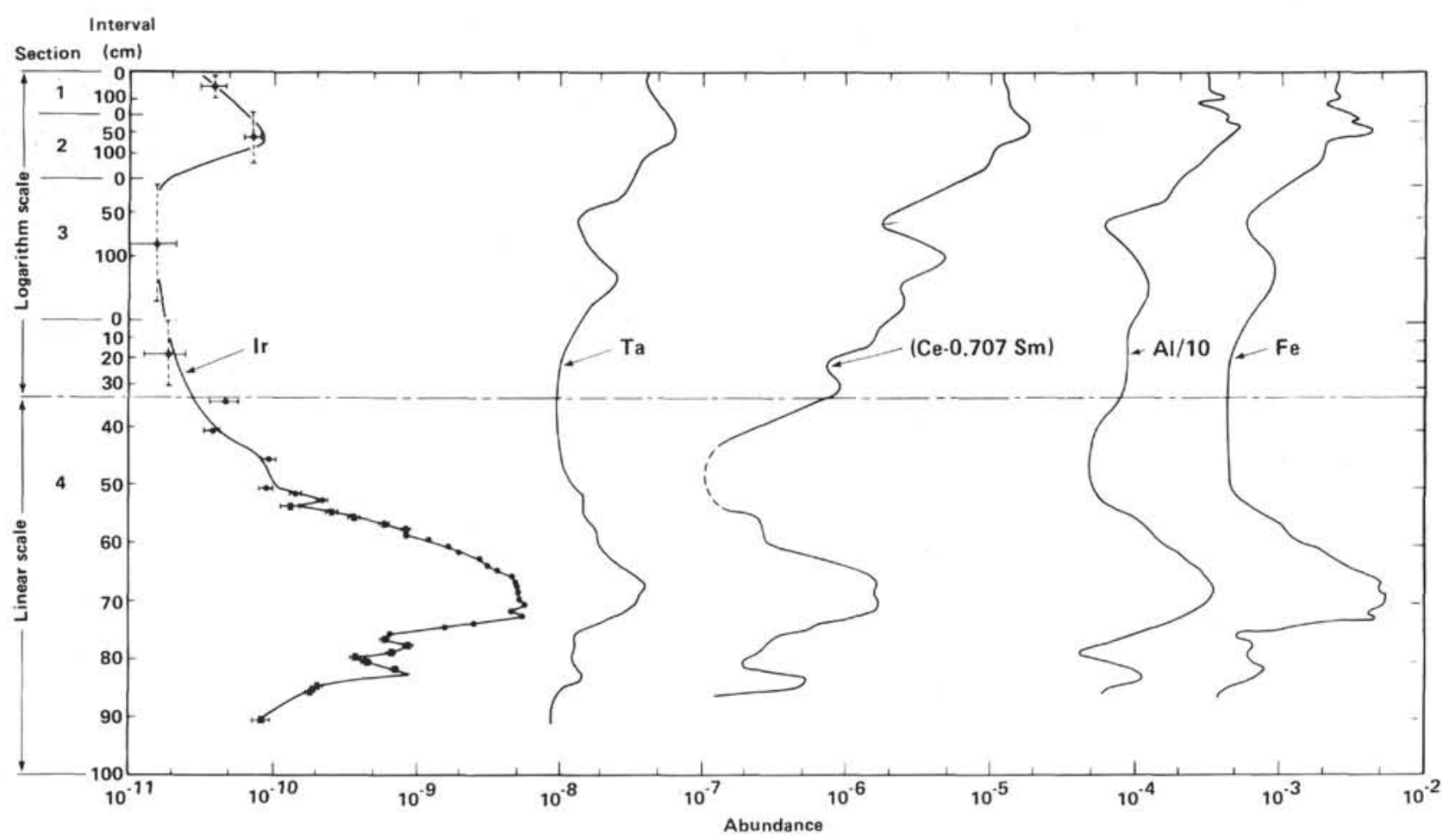

Figure 3. Abundances of $\mathrm{Ir}, \mathrm{Ta},(\mathrm{Ce}-0.707 \mathrm{Sm}), \mathrm{Al} / 10$ and $\mathrm{Fe}$ as a function of stratigraphic position in Core 577B-1, Shatsky Rise.

\section{CONCLUSION}

The Ir anomaly predicted by the asteroid-impact theory was found in the Cretaceous/Tertiary boundary region of Hole $577 \mathrm{~B}$ and its magnitude was $61 \mathrm{ng} / \mathrm{cm}^{2}$. The sharp rise of the main Ir peak occurs within $2 \mathrm{~cm}$ of the Cretaceous/Tertiary boundary as defined by nannoplankton (Monechi, this volume). Five other small Ir peaks as well as an elevated tail are also observed near the main Ir peak. Two of the small peaks are probably related to changes in the $\mathrm{CaCO}_{3}$ deposition rate, while two others and the elevated tail may be due to perturbations of the main peak. One small Ir peak remains unexplained. Siderophile element $(\mathrm{Cr} / \mathrm{Ir}$ and $\mathrm{Ni} / \mathrm{Ir})$ ratios are somewhat different from chondritic values in the Cretaceous/Tertiary region, but this may be due to loss of elements in the oxidizing marine environment.

\section{ACKNOWLEDGMENTS}

We are grateful to Audrey Wright and Amy Altman for their assistance in obtaining the samples, to Audrey Wright for her help in editing the manuscript, and to Charles J. Orth and Frank T. Kyte for reviewing the manuscript. We thank Tek Lim and his staff at the Berkeley Triga Reactor for the neutron irradiations. We also appreciate the assistance of Raphael Zamora and Jon Bechtel in sample preparation and data reduction. Jonathan Stebbins was most helpful in the development of the high-temperature Ir separations.

Funding for this work was provided by the Director, Office of Energy Research, Office of Basic Sciences Division of Engineering, Mathematical and Geosciences of U.S. Department of Energy under Contract No. DE-AC03-76SF00098, the U.S. National Science Foundation under Grant EAR-81-15858, and the National Aeronautics and Space Administration Ames Research Center under Contract A-71683B. DSDP samples were provided through the assistance of the National Science Foundation.

\section{REFERENCES}

Alvarez, L. W., Alvarez, W., Asaro, F., and Michel, H. V., 1980. Extraterrestrial cause for the Cretaceous-Tertiary extinction. Science, 208:1095-1108.

Alvarez, W., Alvarez, L. W., Asaro, F., and Michel, H. V., 1982a. Iridium anomaly approximately synchronous with terminal Eocene extinctions. Science, 216:868-888. $1982 \mathrm{~b}$. Current status of impact theory for the terminal Cretaceous extinction. Spec. Pap. Geol. Soc. Am., 190:305-315. , 1984. The end of the Cretaceous: Sharp boundary or gradual transition. Science, 223:1183-1186.

Asaro, F., Alvarez, L. W., Michel, H. V., and Alvarez, W., 1982. Letter to Editor. Am. Sci., 70:567-568.

Barker, J. L., and Anders, E., 1968. Accretion rate of cosmic matter from iridium and osmium contents of deep sea sediments. Geochim. Cosmochim. Acta, 32:627-645.

Davis, M., Hut, P., and Muller, R. A., 1984. Extinctions of species by periodic comet showers. Nature, 308:715-717.

Kyte, F., and Wasson, J. T., 1982. A search for iridium anomalies in sediments deposited during the past $70 \mathrm{Ma}$. Lunar Planet. Sci., XIII:411-412. (Abstract)

Masuda, A., Nakamura, N., Tanaka, T., 1973. Fine structures of mutually normalized rare-earth patterns of chondrites. Geochim. Cosmochim. Acta, 37:239-248.

Perlman, I., and Asaro, F., 1969. Pottery analysis by neutron activation. Archaeometry, 11:21-52.

1971. Pottery analysis by neutron activation. In Brill, R. H. (Ed.), Science and Archaeology: Cambridge (M.I.T. Press), pp. 182-195.

Rammensee, W., and Palme, H., 1982. "Metal-silicate extraction technique for the analysis of geological and meteoritic samples." J. Radioanal. Chem., 71:401-418.

Whitmire, D. P., and Jackson, A. A., IV, 1984. Are periodic mass extinctions driven by a distant solar companion? Nature, 308: 713-715.

Date of Initial Receipt: 30 January 1984

Date of Acceptance: 6 June 1984 